\title{
openheart Vascular comorbidities worsen prognosis of patients with heart failure hospitalised with COVID-19
}

\author{
Jacob Mok (i) , ${ }^{1}$ Juan Carlos Malpartida, ${ }^{1}$ Kimberly O'Dell, ${ }^{1}$ Joshua Davis, ${ }^{2}$ \\ Cuilan Gao, ${ }^{3}$ Harish Manyam ${ }^{2,4}$
}

\begin{abstract}
- Additional supplemental material is published online only. To view, please visit the journal online (http://dx.doi.org/10.
\end{abstract} 1136/openhrt-2021-001668).

To cite: Mok J, Malpartida JC, O'Dell K, et al. Vascular comorbidities worsen prognosis of patients with heart failure hospitalised with COVID-19. Open Heart 2021;8:e01668. doi:10.1136/

openhrt-2021-001668

Received 31 March 2021 Accepted 25 May 2021

\section{Check for updates}

(c) Author(s) (or their employer(s)) 2021. Re-use permitted under CC BY-NC. No commercial re-use. See rights and permissions. Published by BMJ.

${ }^{1}$ Internal Medicine, UTHSC College of Medicine Chattanooga, Chattanooga, Tennessee, USA

${ }^{2}$ Cardiovascular Disease, UTHSC College of Medicine Chattanooga, Chattanooga, Tennessee, USA

${ }^{3}$ Mathematics, The University of Tennessee at Chattanooga, Chattanooga, Tennessee, USA ${ }^{4}$ Heart and Lung Institute, Erlanger Health System, Chattanooga, Tennessee, USA

Correspondence to Dr Jacob Mok; jmok1990@ gmail.com

\section{ABSTRACT}

Background Prior diagnosis of heart failure (HF) is associated with increased length of hospital stay (LOS) and mortality from COVID-19. Associations between substance use, venous thromboembolism (VTE) or peripheral arterial disease (PAD) and its effects on LOS or mortality in patients with HF hospitalised with COVID-19 remain unknown.

Objective This study identified risk factors associated with poor in-hospital outcomes among patients with HF hospitalised with COVID-19.

Methods Case-control study was conducted of patients with prior diagnosis of HF hospitalised with COVID-19 at an academic tertiary care centre from 1 January 2020 to 28 February 2021. Patients with HF hospitalised with COVID-19 with risk factors were compared with those without risk factors for clinical characteristics, LOS and mortality. Multivariate regression was conducted to identify multiple predictors of increased LOS and in-hospital mortality in patients with HF hospitalised with COVID-19. Results Total of 211 patients with HF were hospitalised with COVID-19. Women had longer LOS than men (9 days vs 7 days; $p<0.001)$. Compared with patients without PAD or ischaemic stroke, patients with PAD or ischaemic stroke had longer LOS ( 7 days vs 9 days; $p=0.012$ and 7 days vs 11 days, $p<0.001$, respectively). Older patients (aged 65 and above) had increased in-hospital mortality compared with younger patients (adjusted OR: $1.04 ; 95 \% \mathrm{Cl} 1.00$ to $1.07 ; p=0.036$ ). Prior diagnosis of VTE increased mortality more than threefold in patients with HF hospitalised with COVID-19 (adjusted OR: 3.33; 95\% Cl 1.29 to 8.43; $\mathrm{p}=0.011$ ).

Conclusion Vascular diseases increase LOS and mortality in patients with HF hospitalised with COVID-19.

\section{INTRODUCTION}

The COVID-19 remains a deadly pandemic that is causing significant morbidity and mortality throughout the world. In spite of this, heart failure (HF) remains one of the most common diagnoses for hospital inpatient stays during the pandemic. A recent study showed that hospital LOS and risk of mortality were increased for patients hospitalised with COVID-19 with a prior diagnosis of $\mathrm{HF}$, regardless of HF type. ${ }^{1}$ However, there

\section{Key questions}

What is already known about this subject?

- Prior diagnosis of heart failure (HF) increases length of hospital stay (LOS) and mortality in patients admitted to the hospital for COVID-19.

- Antiplatelet, anticoagulation and statin therapy decreased venous thromboembolism (VTE) in patients admitted for COVID-19.

What does this study add?

- This study showed that patients with COVID-19, HF and VTE had a higher mortality rate than patients with COVID-19 and either HF or VTE or patients with HF and/or VTE who did not have COVID-19.

- This study showed that patients with HF hospitalised with COVID-19 had greater length of stay with prior diagnosis of peripheral arterial disease or ischaemic stroke.

- This study showed that the increased mortality and the LOS were present without significant difference in HF types.

How might this impact on clinical practice?

- Our findings demonstrate clinical relevance by showing supportive evidence for antiplatelet, anticoagulation and statin therapy in patients with $\mathrm{HF}$ hospitalised with COVID-19

is currently limited data on how different types of comorbidities impact the LOS and mortality of patients with HF hospitalised with COVID-19. Furthermore, the effect of substance use, peripheral arterial disease (PAD) and venous thromboembolism (VTE) on LOS and mortality in patients with HF hospitalised with COVID-19 remains unknown. This study analysed clinical characteristics, length of stay (LOS) and in-hospital mortality of patients with HF admitted for COVID-19 at an academic tertiary care centre.

\section{METHODS}

We conducted a retrospective-cohort study on patients aged 18 years or older admitted 
to one of five Erlanger Healthcare System hospitals with COVID-19 and HF from January 2020 to February 2021. Utilising the electronic health records, we confirmed the diagnosis of COVID-19 with positive PCR test and classified HF into HF with preserved ejection fraction (HFpEF), HF with mid-range ejection fraction (HFmrEF) or HF with reduced ejection fraction (HFrEF). The cardiac function was verified through echocardiogram results, and supporting medical history was used for stratification according to the American Heart Association definition of $\mathrm{HF}^{2}$ Patients with left ventricular ejection fraction (LVEF) less than $40 \%, 41 \%$ to $49 \%$ and $50 \%$ and above were classified as HFrEF, HFmrEF and HFpEF, respectively.

Further patient data collection included patient demographics, substance use, diagnoses, LOS, number of cardiac arrests during admission and in-hospital death. Through this manual chart review process, we identified patient factors of age, sex, body mass index (BMI), substance use and comorbidities. We set specific comorbidities related to the cardiopulmonary system as prior diagnosis of atrial fibrillation (AF), asthma, coronary artery disease (CAD), chronic bronchitis (CB), chronic kidney disease (CKD), chronic obstructive pulmonary disease (COPD), diabetes mellitus (DM), hyperlipidaemia (HLD), hypertension (HTN), obstructive sleep apnea (OSA), PAD, ischaemic stroke (stroke) and VTE, and substance use. VTE included pulmonary embolism (PE) and deep venous thrombosis. Substance use included tobacco, alcohol and intravenous drug use (IVDU).

After collecting data, we used $\mathrm{R}$ statistical software to determine which risk factors were associated with LOS or mortality. Risk factors (age, sex, BMI, tobacco use, alcohol use, IVDU, AF, asthma, CAD, CB, CKD, COPD, DM, HLD, HTN, OSA, PAD, stroke and VTE) were set as either categorical or continuous variables depending on the type of statistical tests applied. For categorical variables, the $\chi^{2}$ test was conducted. If the sample size was small, we replaced the $\chi^{2}$ test with the Fisher's exact test. For continuous variables, the t-test or the Whitney's U test was conducted. If the risk factor followed a normal distribution, a t-test was performed with mean and SD. If the risk factor did not follow a normal distribution, the Whitney's U test was performed with median and IQR.

We compared every risk factor to determine if a significant difference exists by HF type. In particular, age, sex, BMI, tobacco use, alcohol use, IVDU, AF, asthma, CAD, CB, CKD, COPD, DM, HLD, HTN, OSA, PAD, stroke and VTE were compared between patients with HFpEF versus patients with HFrEF. Various statistical tests were applied to compare the risk factors accordingly. The t-test was used for difference in age. The Whitney's $U$ test was used for differences in BMI and LOS. $\chi^{2}$ test or Fisher's exact test was used for differences in sex, substance use, comorbidities, number of cardiac arrests and in-hospital death. Risk factors were also evaluated to determine their effects on mortality in patients with HF in this study. All results with a p-value less than 0.05 were considered statistically significant.

We considered risk factors as dichotomous categorical variables when determining whether or not they were related to mortality. Risk factors, age and BMI were converted from continuous to dichotomous categorical variables by designating specific parameters. Patients aged 65 and above (age 65+) were counted precisely, as this is the general age considered senior citizen. We defined obesity as BMI 30 and above. Including these converted categorical variables, we conducted the $\chi^{2}$ test to compare which risk factors were associated with in-hospital mortality. For risk factors with small sample size, we used the Fisher's exact test instead. The corresponding OR, 95\% CI and $\mathrm{p}$ value were calculated for each factor. To determine the effects on mortality from coexistence of multiple factors, we selected age and other risk factors with OR greater than 1 and $p$ values less than 0.2 for univariate predictors of mortality. We designated tobacco, alcohol and IVDU as confounding variables for mortality. With the risk factors that met these criteria and the designated confounders, we performed a multivariate regression to consider their confounding impact when present in same patient. We determined the adjusted OR and respective $p$ value for this process. Because COVID-19 is a virus that affects the cardiopulmonary system, acute respiratory distress syndrome (ARDS), intubation and intensive care unit (ICU) admission rates were obtained with number of patients and percentage for our study. We defined ARDS with the Berlin definition considering arterial oxygen partial pressure to fractional inspired oxygen ratio ( $\mathrm{PaO} 2 / \mathrm{FiO} 2$ ratio), acuity of symptoms within 1 week and bilateral opacification consistent with pulmonary oedema unexplained by cardiac failure or fluid overload. We designated degree of ARDS severity as mild, moderate and severe with $\mathrm{PaO} 2 / \mathrm{FiO} 2$ ratio of 200-300, 100-200 and less than 100, respectively.

To maintain consistency in our study, we selected identical risk factors to test for mortality and LOS. To determine the effects of risk factors on LOS of our study population (patients with HF hospitalised with COVID19), we calculated the median LOS for patients with and without each risk factor. Respective IQRs per median were also computed. We conducted the Whitney's U test with the resulting medians to determine whether a significant difference exists between patients with the risk factor versus patients without the risk factor. To determine the effects on LOS from co-xistence of multiple factors, we selected risk factors with $\mathrm{p}$ value less than 0.2. Risk factors with any identical IQR for absence or presence were excluded. We determined the coefficient, 95\% CI and respective $p$ values to complete the LOS multivariate analysis. $P$ value less than 0.05 was considered statistically significant for risk factor impact on LOS.

On completion of the mortality and LOS analysis for patients with prior HF hospitalised with COVID19 , we widened the scope of our research to compare among other patient population. After discovering 
the increased mortality from prior diagnosis of VTE in patients with HF hospitalised with COVID-19, we further investigated to determine whether patients with VTE, HF and COVID-19 had higher mortality rates than patients with absence of any of the three diagnoses. More specifically, we compared mortality among patients with COVID-19, HF and VTE versus patients with COVID-19 and HF, patients with COVID-19 and VTE without HF, patients with HF and VTE who did not have COVID-19 and patients with HF who did not have COVID-19 or VTE.

\section{RESULTS}

Our study population consisted of patients with a prior diagnosis of HF admitted for COVID-19. A total of 215 patients were included in the study. Of these patients, 119 (54.5\%) had HFpEF and 96 (45.5\%) had HFrEF. Table 1 outlines the clinical characteristics of our study population stratified by HF types. Compared with patients with HFrEF, patients with HFpEF were significantly more overweight or obese, more likely to have a stroke and predominantly women. Furthermore, there was no significant difference in age, substance use, comorbidities

Table 1 Clinical characteristics and outcomes of study population according to heart failure types

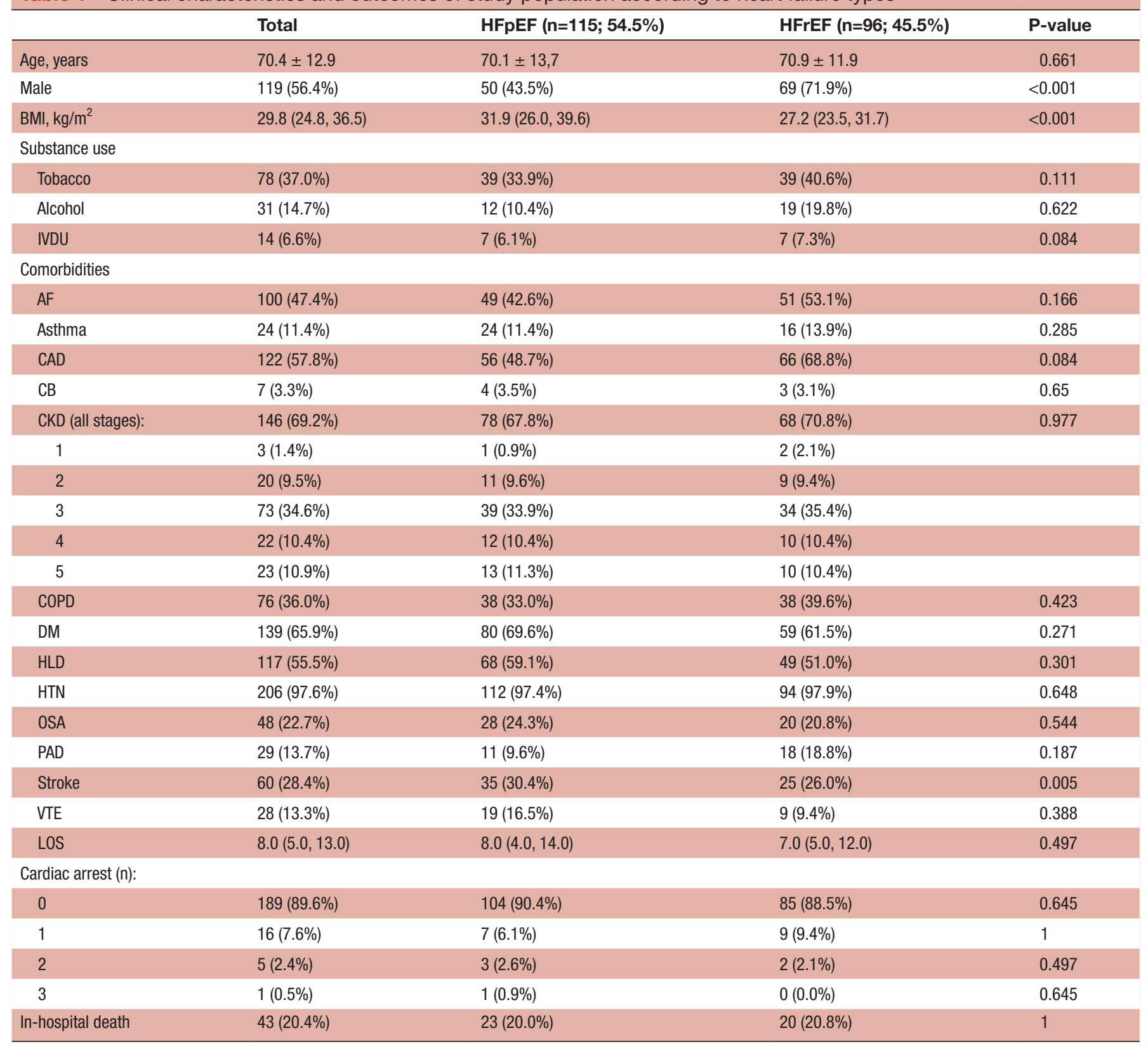

Values are $\mathrm{n}(\%)$, mean \pm standard deviation, median (interquartile range).

$\mathrm{AF}$, atrial fibrillation; $\mathrm{BMI}$, body mass index; $\mathrm{CAD}$, coronary artery disease; $\mathrm{CB}$, chronic bronchitis; CKD, chronic kidney disease; COPD, chronic obstructive pulmonary disease; DM, diabetes mellitus; HF, heart failure; HFmrEF, HF with mid-range ejection fraction; HFpEF, HF with preserved ejection fraction ; HLD, hyperlipidaemia; HTN, hypertension; IVDU, intravenous drug use; OSA, obstructive sleep apnea; PAD, peripheral arterial disease; Stroke, ischemic stroke; VTE, venous thromboembolism including deep venous thrombosis and pulmonary embolism; yrs, years. 


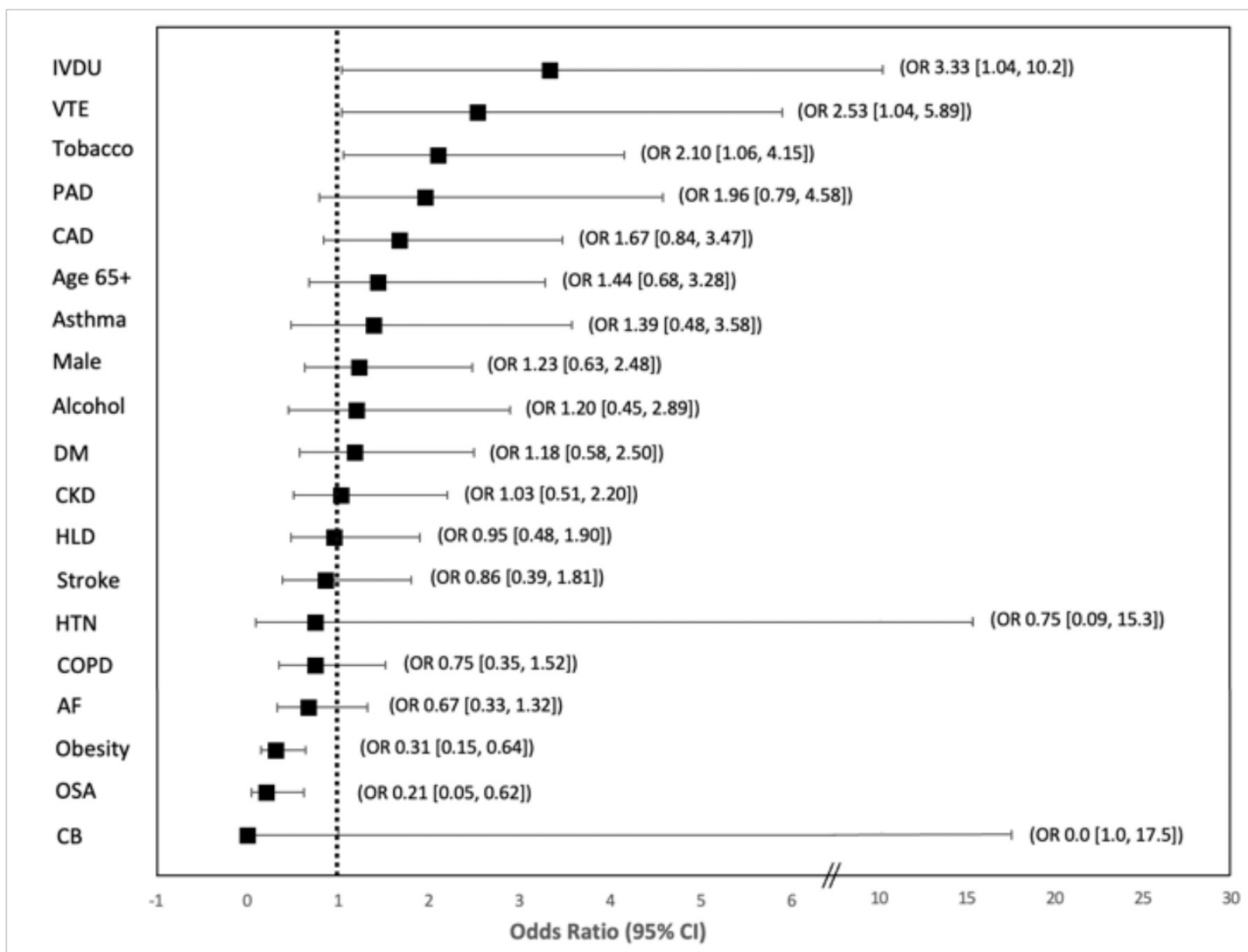

Figure 1 Forest plot for the effect of risk factors on in-hospital mortality for all patients with HF admitted for COVID-19. HF, heart failure; IVDU, intravenous drug use; VTE, deep venous thrombosis and pulmonary embolism; PAD, peripheral arterial disease; CAD, coronary artery disease; Age 65+, age greater than or equal to 65; DM, diabetes mellitus; CKD, chronic kidney disease; HLD, hyperlipidaemia; HTN, hypertension; COPD, chronic pulmonary obstructive disease; AF, atrial fibrillation; OSA, obstructive sleep apnea; CB, chronic bronchitis;

(excluding stroke), LOS, number of cardiac arrest(s) or in-hospital death between patients with HFpEF and patients with HFrEF.

Risk factors were analysed to determine any association with mortality in our study population. Figure 1 summarises the effect of each risk factor on in-hospital mortality. IVDU (OR: 3.33; 95\% CI 1.04 to 10.2; $\mathrm{p}=0.035$ ), prior diagnosis of VTE (OR: 2.53; 95\% CI 1.04 to 5.89; $\mathrm{p}=0.035$ ) and tobacco use (OR: 2.10; 95\% CI 1.06 to 4.15 ; $\mathrm{p}=0.033$ ) showed significant increase in risk of in-hospital death. Obesity (OR: $0.31 ; 95 \%$ CI 0.15 to $0.64 ; \mathrm{p}=0.012$ ) and OSA (OR: $0.21 ; 95 \%$ CI 0.05 to $0.62 ; \mathrm{p}=0.012$ ) showed significant decrease in risk of in-hospital death. The effect of $\mathrm{CB}$ on mortality remains indeterminate given $100 \%$ survival in our study (OR: $0.0 ; 95 \%$ CI 1.0 to 17.5 ; $\mathrm{p}=0.986$ ). Post multivariate regression results showed age $65+$ as a significant risk factor (adjusted OR: 1.04; 95\% CI 1.00 to $1.07 ; \mathrm{p}=0.036)$ and increased mortality risk for VTE (adjusted OR: 3.33; 95\% CI 1.29 to 8.43 ; $\mathrm{p}=0.011$ ). These findings are demonstrated in online supplemental tables 1 and 2. Further analysis was performed to compare the percentages of in-hospital deaths by the type of vascular disease present in our study population. Figure 2 represents the percentages of deaths in patients with prior diagnosis of VTE, PAD, CAD or stroke. In our study, patients with HF admitted for COVID-19 with prior diagnosis of VTE, PAD, CAD or stroke faced in hospital death by $36 \%, 31 \%, 24 \%$ and $18 \%$, respectively. Patients

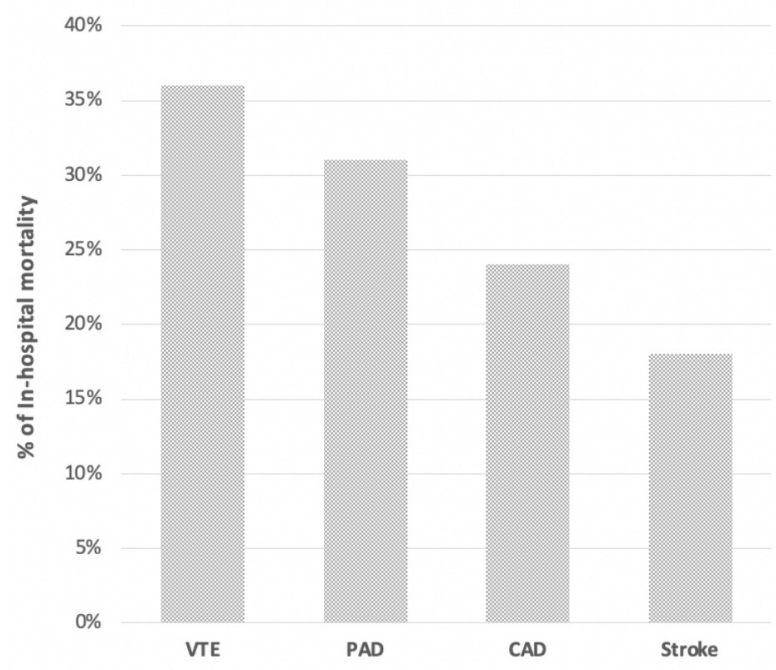

Figure 2 In-hospital mortality of HF patients stratified by type of vascular disease. HF, heart failure; VTE,venous thromboembolism; PAD, peripheral arterialdisease; CAD, coronary artery disease. 
Table 2 Length of hospital stay for patients with HF admitted for COVID-19 by risk factors

\begin{tabular}{lccl}
\hline Risk factors & LOS (+) & LOS (-) & P value \\
\hline Age $\geq 65$ & $8(5,12)$ & $8(5,14)$ & 0.439 \\
Male & $7(4,12)$ & $9(5,14)$ & $\mathbf{0 . 0 3 6}$ \\
\hline Obesity $(\mathrm{BMI} \geq 30)$ & $8(5,12)$ & $8(5,13)$ & 0.810 \\
\hline Substance use & & & \\
\hline Tobacco & $7(4,13)$ & $8(5,13)$ & 0.507 \\
\hline Alcohol & $10(5.5,14.5)$ & $8(5,12)$ & 0.260 \\
\hline IVDU & $14(11.3,19.3)$ & $7(4.5,12)$ & $\mathbf{0 . 0 0 2}$ \\
Comorbidities & & & \\
\hline AF & $8(5,12)$ & $8(4.5,14)$ & 0.859 \\
\hline Asthma & $13(9,20.8)$ & $7(4,12)$ & $\mathbf{0 . 0 0 1}$ \\
\hline CB & $5(5,11)$ & $8(5,13)$ & 0.472 \\
\hline CAD & $8(5,13)$ & $8(4,13)$ & 0.817 \\
\hline CKD & $8(4,12)$ & $7(5,14)$ & 0.716 \\
\hline COPD & $7(5,11)$ & $8(5,14)$ & 0.247 \\
\hline DM & $9(4.5,14)$ & $7(5,11.5)$ & 0.428 \\
\hline HLD & $7(5,12)$ & $9(5,14)$ & 0.203 \\
\hline HTN & $8(5,13)$ & $12.5(4,20.5)$ & 0.901 \\
\hline OSA & $10(5,14)$ & $7.5(5,13)$ & 0.501 \\
\hline PAD & $9(6,16)$ & $7(5,12)$ & $\mathbf{0 . 0 1 *}$ \\
\hline Stroke & $11(5.75,17.5)$ & $7(4,11)$ & $\mathbf{0 . 0 0 2}$ \\
\hline VTE & $11.5(5,14.3)$ & $7(5,12)$ & 0.127 \\
\hline
\end{tabular}

Values are represented by median (interquartile range) number of days hospitalised.

${ }^{*}$ Adjusted after multivariate linear regression.

$\mathrm{AF}$, atrial fibrillation; $\mathrm{BMI}$, body mass index; $\mathrm{CAD}$, coronary artery disease; CB, chronic bronchitis; CKD, chronic kidney disease; COPD, chronic obstructive pulmonary disease; DM, diabetes mellitus; HF, heart failure; HLD, hyperlipidaemia; IVDU, intravenous drug use;

Stroke, ischemic stroke; VTE, venous thromboembolism including deep venous thrombosis and pulmonary embolism.

in our study were evaluated for ARDS, intubation and ICU admission rates. Online supplemental table 5 shows that patients with HF hospitalised with COVID-19 who faced in-hospital death were diagnosed with ARDS, admitted to the ICU and intubated $83.7 \%, 80 \%$ and $87.5 \%$ of the time, respectively.

Risk factor association with LOS was also compared in our study. Table 2 outlines the effect of each risk factors on hospital LOS. IVDU, asthma, stroke and PAD significantly increased LOS in our study population. The median LOS for our study population with and without IVDU was 14 days (IQR: 11 to 19 days) and 7 days (IQR: 4.5 to 12 days), respectively. The median LOS for patients with and without asthma was 13 days (IQR: 9 to 21 days) and 7 days (IQR 4 to 12 days), respectively. The median LOS for patients with and without stroke was 11 days $(6$ to 18 days) and 7 days (4 to 11 days), respectively. The median LOS for patients with and without PAD was 9 days (IQR: 6 to 16 days) and 7 days (IQR: 5 to 12 days), respectively. Interestingly, women had significantly longer LOS than men. The median LOS for women and men was 9 days (IQR: 5 to 14 days) and 7 days (IQR: 4 to 12 days), respectively. Selection for multivariate regression and resulting values are demonstrated in online supplemental tables 3 and 4 .

Figure 3 shows in-hospital mortality rates among patients with COVID-19, HF,and VTE; COVID-19 and HF without VTE; COVID-19 and VTE without HF; HF and VTE without COVID-19 and HF without COVID-19 or VTE as $36 \%, 17 \%, 12 \%$ and $12 \%$, respectively. Additionally, figure 3 shows that prior diagnosis of HF in patients hospitalised for COVID-19 increases in-hospital mortality to nearly two times, as shown by the mortality rate in patients with COVID-19 and HF versus COVID-19 with no $\mathrm{HF}$ to be $20 \%$ and $11 \%$, respectively.

\section{DISCUSSION}

HF is a condition that reduces immunity. Through the increase of tumour necrosis factor alpha (TNF-a) and decrease of IL-10, HF promotes an inflammatory process that intensifies the severity of infectious illness. ${ }^{3}$ COVID-19

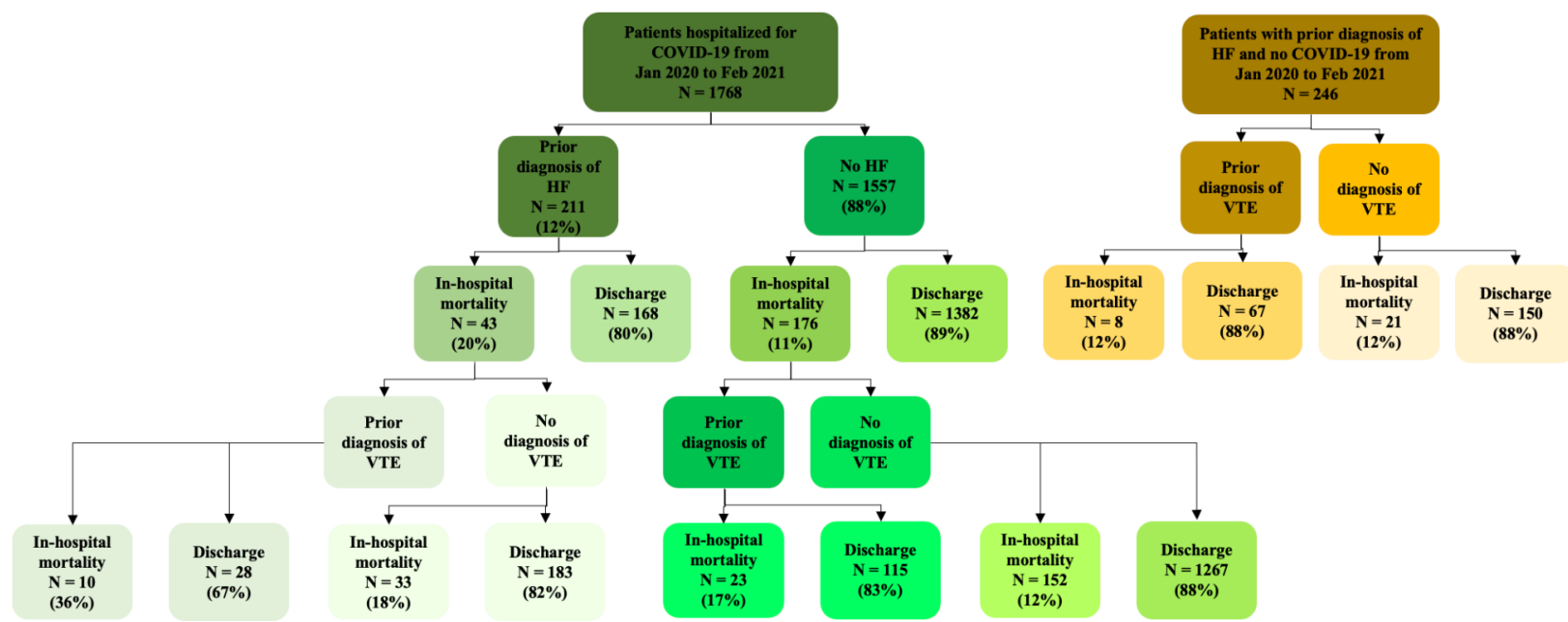

Figure 3 Analysis of hospitalisation for patients based on diagnoses of COVID-19, VTE, HF. VTE, venous thromboembolism; HF, heart failure. 
was not an exception to the infections that exacerbate patients with HF. Our study revealed that $20 \%$ of patients with prior HF hospitalised with COVID-19\% and $11 \%$ of patients with no HF hospitalised with COVID-19 faced in-hospital death. Compared with a previous study that tallied in-hospital deaths in 1000 hospitals, this percentage was vastly greater than $4.5 \%$ of patients admitted without COVID-19 or HF and consistent with $24 \%$ of the patients hospitalised with COVID-19 with prior $\mathrm{HF}^{4}$ Our study was also congruent for no significant difference in mortality between HF types with a previous study that indicated two times the risk of mortality in patients with COVID-19 with $\mathrm{HF}$ versus without $\mathrm{HF}^{1}{ }^{1}$ We designated comorbidities AF, HTN, DM, CKD, COPD, OSA, CB, asthma, HLD, stroke, CAD, PAD and VTE to investigate their effects on LOS and mortality of patients with HF admitted with COVID-19. Through this process, we discovered that the diagnosis of obesity resulted in three times less risk of death than without obesity (OR $0.31 ; 95 \%$ CI 0.15 to 0.64 ; $\mathrm{p}=0.002$ ). This finding was consistent with a meta-analysis study that revealed being overweight and obese were protective factors against cardiovascular mortality and all-cause mortality in patients with $\mathrm{HF}^{5}$ We also found that prior diagnosis of VTE resulted in greater than three times the risk of death in patients with COVID-19 with HF than without VTE (OR 3.33; 95\% CI 1.29 to 8.43; $\mathrm{p}=0.011$ ). More specifically, our study revealed that one of three patients with HF, VTE and COVID-19 had in-hospital death and one of eight patients with HF, VTE and no COVID-19 had in-hospital death.

The mechanism of how COVID-19 increases mortality in patients with HF and VTE remains unknown. However, understanding the pathophysiology of HF and COVID-19 helps develop a conjecture about the increased mortality in patients with HF, COVID-19 and VTE as reflected in our study. HF promotes thrombogenesis through endothelial dysfunction and inflammation, as noted by decreased endothelial nitric oxide synthase activity and increased IL-1, IL-6, TNF-a, soluble thrombomodulin, circulating endothelial cells, $\mathrm{C}$ reactive protein, angiopoietin-2, tissue factor, E-selectin and von Willebrand factor $(\mathrm{vWF}){ }^{6}$ Moreover, HF reduces haemodynamic performance, exacerbating the ability to cope with increased cardiac output required in the setting of inflammation due to infections. ${ }^{3}$ COVID-19-induced ARDS showed significantly higher platelets, fibrinogen and thrombotic complications than non-COVID-19 ARDS. ${ }^{7}$ Furthermore, COVID-19 patients with ARDS had more than five times incidence of PE with very high levels of vWF antigen. ${ }^{7}$

Perhaps, the increased vWF in both HF and COVID-19 provides an explanation to the increased mortality of patients with COVID-19, HF and VTE versus patients with COVID-19 and VTE, HF and VTE or COVID-19 without VTE. The percentage of death for patients with COVID-19, HF and VTE in our study was $35.7 \%$ while the percentage of death for patients with COVID-19 and VTE, HF and VTE and COVID-19 without VTE was approximately $17 \%, 12 \%$ and $12 \%$, respectively. Increased mortality of patients inclusive of all three conditions, VTE, HF and COVID-19, compared with patients with an exclusion of any of these conditions demonstrate that thrombotic parameters from combination of $\mathrm{HF}$ and COVID-19 warrant investigation. Supplemental to these findings, studies showed that patients with COVID-19 and HF had two times the greater risk of death compared with patients with COVID-19 and no HF while patients with COVID-19 and VTE also had two times the greater risk of death compared with patients with COVID-19 and no VTE. ${ }^{18}$ Studies also revealed that VTE increased the incidence of $\mathrm{HF}$ while $\mathrm{HF}$ also increased the incidence of VTE, highlighting the detrimental cycle of HF in association with VTE. ${ }^{6}$ These relations of COVID-19, HF and VTE underscore the importance of optimal medical management for morbidity and mortality reduction.

The exact treatment regimen for acute COVID-19 infection is yet to be established. The treatment decisions become even more abstruse in the setting of a coexistent HF. All patients in this study had prior HF and received anticoagulation. Patients with deep VTE, pulmonary embolus or AF with CHA2DS2-VASc score greater than or equal to 2 received various types of anticoagulation at treatment dose while patients without these conditions received heparin or enoxaparin at thromboprophylaxis dose. All patients in this study with CAD, PAD and ischaemic stroke received statin and antiplatelet therapy with aspirin or other P2Y12 inhibitors. Our study also showed patients with HF admitted for COVID-19 had longer LOS with diagnosis of PAD or ischaemic stroke ( 7 days vs 9 days; $\mathrm{p}=0.012$ and 7 days vs 11 days, $\mathrm{p}<0.001$; respectively). A multihospital retrospective study revealed that antiplatelet and statin use decreased VTE or mortality by $35 \%$ and $45 \%$, respectively in patients with COVID- $19 .^{10}$ Another study also showed that patients with COVID-19 receiving therapeutic anticoagulation prior to admission had three times less risk of developing VTEs and did not significantly suffer more from all-cause death than patients without anticoagulation. ${ }^{8}$ Furthermore, heparin use during admission was associated with two times as less mortality in patients with HF with COVID-19. ${ }^{11}$

\section{Limitations}

The use of electronic health records with International Classification of Diseases-9/10 (ICD-9/10) codes for HF potentially misclassified some patients per HF type. ICD-9/10 codes with congestive HF, concurrent HFpEF and HFrEF and HF without further specifications required sorting to appropriate $\mathrm{HF}$ types. To minimise these classification errors, we manually ascertained HF type through comprehensive review of documentations, echocardiograms and medication list. HFmrEF was not analysed as a separate entity due to inadequate number. In efforts to ensure the absence of HFmrEF category does not derange our study, we performed analyses with HFmrEF classified as HFpEF and without HFmrEF. The significance of the results did not vary when comparing 
HFmrEF as HFpEF or excluding the HFmrEF patient data sets altogether.

Details to prior diagnosis of VTE such as onset, provocation and recurrence were inconclusive to determine whether anticoagulation before admission was indicated. Moreover, $36 \%$ of patients with prior diagnosis of VTE were on anticoagulation before hospitalisation. Patients with VTE on anticoagulation and patients with VTE not on anticoagulation had mortality rate of $30 \%$ and $33 \%$, respectively, in our study. Future study focusing on anticoagulation in patients with prior HF and VTE hospitalised for COVID-19 would help solidify our findings and optimal therapy.

Interestingly, our study showed OSA to be a protective factor against mortality (OR $0.21 ; 95 \%$ CI 0.05 to 0.62 ; $\mathrm{p}=0.012)$. However, the significance of OSA for mortality reduction remains questionable in our study given its low prevalence in our study population. We identified risk factors for OSA including BMI, HTN, smoking, asthma, sex (male) and DM. HTN, DM, sex (male), obesity, tobacco use and asthma were present in $98 \%, 66 \%, 56 \%$, $49 \%, 36 \%$ and $11 \%$ of our patients, respectively. Given the predominant existence of OSA risk factors and only three patients with OSA with in-hospital death, we predict that OSA is under diagnosed in our study population. Obesity was also reflected to be a protective factor against mortality (OR: $0.31 ; 95 \%$ CI 0.15 to $0.64 ; \mathrm{p}=0.012$ ). However, we found that patients with normal BMI in our study had lower LVEF than overweight or obese patients with average LVEF for BMI less than 25 versus greater than or equal to 25 at $40 \%$ and $48 \%$, respectively (t-test $\mathrm{p}<0.00001)$. The difference between the two groups was even more evident in those who faced in-hospital mortality, as patients with BMI less than 25 versus greater than or equal to 25 had LVEF $36 \%$ and $49 \%$, respectively (t-test $\mathrm{p}=0.004$ ). These findings suggest that further study is warranted to investigate the association of obesity with LVEF and arguably undermine obesity as an independent protective factor against mortality. Future studies should also explore why female patients with HF hospitalised with COVID-19 had significantly higher LOS than male patients with HF hospitalised with COVID-19.

\section{CONCLUSION}

Our study revealed that patients with prior HF and VTE admitted for acute COVID-19 infection faced greater than threefold increased risk of in-hospital death versus patients without VTE. Our study also showed the increased hospital LOS in patients with stroke and PAD. Standardised antithrombotic treatment for patients with HF and COVID-19 is inconclusive. With several studies demonstrating mortality decrease with antiplatelet, anticoagulation and statin therapy for patients with HF hospitalised with COVID-19, clinicians could possibly consider a lower threshold in initiating antithrombotic and statin therapy if findings are confirmed in other populations.

Acknowledgements The authors would like to thank Dr. Stephen Adams (Erlanger Health Systems) for data acquisition and the research department of the University of Tennessee College of Medicine at Chattanooga for coordinating assistance with the biostatistician at the mathematics department of the University of Tennessee at Chattanooga. The authors would like to thank Dr. Jonathan Pumilia and Dr. Anne Mainardi for critically reviewing the manuscript.

Funding The authors have not declared a specific grant for this research from any funding agency in the public, commercial or not-for-profit sectors.

Competing interests None declared.

Patient consent for publication Not required.

Ethics approval University of Tennessee at Chattanooga Institutional Review Board approved this study. The approval study number is 21-011.

Provenance and peer review Not commissioned; externally peer reviewed.

Data availability statement All data relevant to the study are included in the article or uploaded as supplemental information. Others can reuse and share our data with others as long as they credit and cite us, but not for commercial uses.

Open access This is an open access article distributed in accordance with the Creative Commons Attribution Non Commercial (CC BY-NC 4.0) license, which permits others to distribute, remix, adapt, build upon this work non-commercially, and license their derivative works on different terms, provided the original work is properly cited, appropriate credit is given, any changes made indicated, and the use is non-commercial. See: http://creativecommons.org/licenses/by-nc/4.0/.

ORCID iD

Jacob Mok http://orcid.org/0000-0001-9748-7692

\section{REFERENCES}

1 Alvarez-Garcia J, Lee S, Gupta A, et al. Prognostic Impact of Prior Heart Failure in Patients Hospitalized With COVID-19. J Am Coll Cardiol 2020;76:2334-48.

2 Yancy CW, Jessup M, Bozkurt B. ACCF/AHA guideline for the management of heart failure. J Am Coll Cardiol 2013;2013:e147-239.

3 Bader F, Manla Y, Atallah B, et al. Heart failure and COVID-19. Heart Fail Rev 2021;26:1-10.

4 Bhatt AS, Jering KS, Vaduganathan M, et al. Clinical Outcomes in Patients With Heart Failure Hospitalized With COVID-19. JACC Heart Fail 2021;9:65-73.

5 Oreopoulos A, Padwal R, Kalantar-Zadeh K, et al. Body mass index and mortality in heart failure: a meta-analysis. Am Heart $J$ 2008;156:13-22.

6 Dean SM, Abraham W. Venous thromboembolic disease in congestive heart failure: venous thromboembolic disease in congestive heart failure. Congestive Heart Failure 2010;16:164-9.

7 Helms J, Tacquard C, Severac F, et al. High risk of thrombosis in patients with severe SARS-CoV-2 infection: a multicenter prospective cohort study. Intensive Care Med 2020;46:1089-98.

8 Malas MB, Naazie IN, Elsayed N, et al. Thromboembolism risk of COVID-19 is high and associated with a higher risk of mortality: a systematic review and meta-analysis. EClinicalMedicine 2020;29:100639.

9 Shantsila E, Lip GYH. Thrombotic complications in heart failure: an underappreciated challenge. Circulation 2014;130:387-9.

10 Giannis D, Barish MA, Goldin M, et al. Incidence of venous thromboembolism and mortality in patients with initial presentation of COVID-19. J Thromb Thrombolysis 2021;51:897-901.

11 Tomasoni D, Inciardi RM, Lombardi CM, et al. Impact of heart failure on the clinical course and outcomes of patients hospitalized for COVID-19. Results of the Cardio-COVID-Italy multicentre study. Eur $J$ Heart Fail 2020;22:2238-47. 